\title{
Effects of sex on sleep-disordered breathing in adolescents
}

\author{
M.A. Fuentes-Pradera*, Á. Sánchez-Armengol*, F. Capote-Gil*, E. Quintana-Gallego*, \\ C. Carmona-Bernal*, J. Polo", F. Delgado-Moreno", J. Castillo-Gómez*
}

Effects of sex on sleep-disordered breathing in adolescents. M.A. Fuentes-Pradera, A. Sánchez-Armengol, F. Capote-Gil, E. Quintana-Gallego, C. Carmona-Bernal, J. Polo, F. Delgado-Moreno, J. Castillo-Gómez. (C) ERS Journals Ltd 2004.

ABSTRACT: This study was conducted to determine the influence of puberty on features of sleep-disordered breathing (SDB) in adolescents.

The study was performed in a general population sample of 226 adolescents of both sexes (aged 11-19 yrs) recruited from the secondary school population of the city of Seville, Spain. Subjects were divided into two groups: 1) postpubertal, i.e. females who had undergone menarche and males in whom axillary hair development or peak height velocity had occurred $>1$ yr before the study; and 2) peripubertal, i.e. females who had not undergone menarche and males who had not developed axillary hair nor reached peak height velocity, or subjects in whom these pubertal changes had appeared $<1 \mathrm{yr}$ before the study. All subjects answered a questionnaire on SDB and underwent overnight cardiorespiratory polygraphy.

There were 50 males and 40 females (mean \pm SD age 13.5 $\pm 1.2 \mathrm{yrs}$ ) in the peripubertal group, and 54 males and 82 females (age 16.3 $\pm 1.7 \mathrm{yrs}$ ) in the postpubertal group. Males exhibited significantly higher neck circumference/height index and waist/hip index than females in both the peripubertal and postpubertal groups. In the postpubertal group, snoring and polygraphic alterations (respiratory events and oximetric parameters) were significantly more frequent in males than in females. Postpubertal adolescents showed sex differences in clinical and polygraphic parameters that were not observed at earlier pubertal stages.

These findings support the influence of sex hormones on sex differences in sleepdisordered breathing.

Eur Respir J 2004; 23: 250-254.
*Medical-Surgical Unit of Respiratory Diseases and \#Service of Otorhinolaryngology, Virgen del Rocío University Hospital, and Dept of Statistics, Seville University, Seville, Spain.

Correspondence: M.A. Fuentes-Pradera, Unidad Médico Quirúrgica de Enfermedades Respiratorias, Hospital Universitario Virgen del Rocío, Manuel Siurot s/n, E-41013 Sevilla, Spain.

Fax: 34955012199

E-mail: mangeles2001@jazzfree.com

Keywords: Adolescence cardiorespiratory polygraphy sleep-disordered breathing

Received: February 272003

Accepted after revision: September 42003

This study was supported by grants $38 / 96$ and 247/97 from the Health Services (Government of Andalusia, Seville, Spain) and the RESPIRA Research Network (RTIC 03/11), Health Institute "Carlos III", Madrid, Spain.
Sleep-disordered breathing (SDB) includes a broad range of symptoms, from slight snoring to severe cases of obstructive sleep apnoea syndrome. Population-based studies have linked even mild SDB among adults with a higher number of traffic accidents, cardiovascular disease and other adverse health outcomes [1-3]. SDB has been assumed to be a condition associated primarily with males. In clinical samples, the ratio of males to females for prevalence of SDB has been considered to be $\sim 10: 1[4,5]$. In general population samples, it has been shown that sleep apnoea occurs more frequently than assumed in females. Most estimates of the male/female ratio in the general public are in the region of $2: 1$ or $3: 1$ [6-8]. The reason for this sexual disparity is not fully understood; however, it has been suggested that the risk factors and mechanisms for the development of SDB, particularly in both the structure and function of the upper airway, may differ between males and females [9-13]. Sex hormones have also been thought to influence the development of obstructive sleep apnoea. It has been shown that menopause is a risk factor for SDB and that hormone replacement therapy modifies this risk [14]. Conversely, treatment with testosterone has been reported to cause obstructive sleep apnoea in males [15]. In addition, a case of a nonobese female with clinically significant obstructive sleep apnoea and a benign testosterone-producing ovarian tumour that resolved after successful removal of the tumour, along with normalisation of testosterone levels, has been reported [16].
SDB in childhood has clinical, diagnostic and therapeutic characteristics that are different from those found in adults [17]; however, sex differences in the prevalence of SDB in the paediatric age group have not been observed [18, 19]. Alterations in upper airway morphology at puberty may play a significant role in the development of SDB in early adulthood [20]. In the adolescent population, however, there is little information regarding the prevalence and clinical expression of SDB [21]. The present study was, therefore, performed to determine the influence of puberty on clinical, anthropometric and polygraphic features related to SDB in a general population sample of adolescents recruited from the secondary school population of the city of Seville, Spain.

\section{Methods}

A cross-sectional study was carried out in a general population sample of 226 adolescents of both sexes aged 11-19 yrs. In order to obtain a representative sample of the general population of adolescents, a full official directory of the 220 public and private secondary schools in the city of Seville, Spain, was obtained. A total of 32 schools were chosen randomly using a table of aleatory numbers. In each school, all the subjects from the same classroom were contacted. No inclusion or exclusion criteria based on the absence or presence of previous or underlying diseases were 
established. The study was approved by the science and education department of the autonomous government of Andalusia. Written informed consent was obtained from adolescents who voluntarily agreed to take part and from their parents or legal guardians. The study included the administration of a questionnaire for the investigation of SDB symptoms, measurement of anthropometric parameters and overnight home polygraphy.

The questionnaire consisted of 82 items (grouped into demographics, salient features of family and personal history, school performance, and nocturnal and daytime symptoms suggestive of SDB), the details of which have been previously described [21]. Symptoms were assessed according to a frequency scale that included the following answers: "unknown", "never", "rarely" ( $\leqslant 1$ event·week $\left.{ }^{-1}\right)$, "sometimes" ( 2 events· week $\left.^{-1}\right)$, and "often" ( $\geqslant 3$ events $\cdot$ week $\left.^{-1}\right)$. Snorers answered "sometimes" or "often" for the question on snoring. Habitual snoring was defined as snoring on $>3$ days $\cdot$ week $^{-1}$. Other symptoms, such as reported sleep apnoeas (stoppage of breathing during sleep observed by parents) were assessed using the same frequency scale and excessive daytime sleepiness according to a three-degree scale: slight, moderate and severe. Anthropometric measurements included weight, height, and neck, waist and hip circumference, as previously described [21]. The body mass index (BMI), defined as the weight in kilograms divided by height squared in metres, and the neck circumference-to-height and waist-to-hip perimeter ratios were calculated. Subjects with a BMI of $\geqslant 28 \mathrm{~kg} \cdot \mathrm{m}^{-2}$ were considered obese.

Subjects were divided into two groups: 1) postpubertal, i.e. females who had undergone menarche and males in whom axillary hair development or peak height velocity had occurred $>1$ yr before the study; and 2) peripubertal, i.e. females who had not undergone menarche and males who had not developed axillary hair nor reached peak height velocity, or subjects in whom these pubertal changes had appeared $<1$ yr before the study.

In all subjects, overnight home polygraphy was performed using a portable ambulatory device (Apnoescreen II; Erich Jaeger Gmbh \& CoKg, Würzburg, Germany), with continuous monitoring of oronasal airflow (thermistor), chest and abdominal respiratory movements (thoracic and abdominal belts), arterial oxygen saturation $\left(\mathrm{Sa}_{\mathrm{a}} \mathrm{O}_{2}\right.$; digital pulse oximetry), cardiac frequency (digital probe), electrocardiography, body position (mercury sensor) and actigraphy (wristband with activity sensor). Analysis was carried out manually. The following parameters were assessed: respiratory events, defined as a decrease in oronasal airflow below a fixed threshold (established as a flow whose intensity corresponds to 35 bytes on the computer graphic scale) for $\geqslant 5 \mathrm{~s}$; oxygen desaturation, defined as a drop in $\mathrm{Sa}_{\mathrm{a}} \mathrm{O}_{2}$ of $\geqslant 4 \%$ for $\geqslant 8 \mathrm{~s}$; and cardiac events, defined as a change in cardiac frequency of $\geqslant 10$ beats. $\min ^{-1}$ for $\geqslant 10 \mathrm{~s}$. Cardiac events were considered to be related to respiratory events and/or desaturations if they occurred simultaneously. Variables analysed were total recording (sleep) time (TRT), TRT in the supine position (TRTsup), respiratory disturbance index (RDI; respiratory events per hour), RDI during TRTsup (RDIsup), oxygen desaturation index (ODI) (number of oxygen desaturations per hour), and total number of cardiac events concurrent with respiratory events and/or oxygen desaturation.

\section{Statistical analysis}

All results are expressed as mean $\pm \mathrm{SD}$. The unpaired twotailed t-test was used for comparison of quantitative variables and the Chi-squared test (with Fisher's exact test when necessary) for comparison of categorical variables between peripubertal and postpubertal adolescents. Analysis of covariance (ANCOVA) was used to compare RDI (independent variable) between males and females in the postpubertal group after adjusting for age, BMI and waist/hip index. Significance was set at $\mathrm{p}<0.05$.

\section{Results}

The target population was made up of 933 subjects, of whom $394(42.2 \%)$ refused to participate in the study. Of the remaining 539, $313(33.5 \%)$ refused to undergo overnight polygraphy but completed a few items of the questionnaire. Thus the study population comprised $226(24.2 \%)$ subjects. There were no differences between the 313 unincluded subjects and the 226 adolescents included in male/female ratio (149/164 versus $104 / 122)$, age (14.5 \pm 2.2 versus $14.9 \pm 2.0 \mathrm{yrs})$, or frequency of snoring (18.4 versus $26.5 \%)$, witnessed apnoeas (4.1 versus $3.1 \%$ ) or sleepiness (33.8 versus $30.1 \%$ ).

Of the 226 included adolescents, aged $14.9 \pm 2.0$ yrs, 104 were male and 122 were female; $19 \pm 8.4 \%$ were obese. At menarche and at onset of development of secondary sexual characteristics, females and males were aged $11.8 \pm 1.1$ and $13 \pm 1.3 \mathrm{yrs}$, respectively. Sixty subjects were classified as snorers and 35 as habitual snorers. The frequency distribution of anthropometric, clinical and polygraphic variables in the study population is shown in table 1 .

\section{Sex differences in the peripubertal group}

The peripubertal group included 90 subjects, 55.5\% male and $43.4 \%$ female, aged $13.5 \pm 1.2$ and $13.1 \pm 0.8 \mathrm{yrs}$, respectively. Menarche or development of secondary sexual characteristics had not occurred in 30 of the adolescents, whereas, in the remaining 60, these changes had occurred within the year prior to the study. There were no differences between males and females in anthropometric, clinical and polygraphic variables, except for significantly higher neck

Table 1.-Frequency distribution of anthropometric, clinical and polygraphic variables in 226 adolescents

\begin{tabular}{lc}
\hline Anthropometric parameters & \\
Age yrs & $14.9 \pm 2.0$ \\
BMI kg.m ${ }^{-2}$ & $21.8 \pm 3.6$ \\
Obesity n $(\%)$ & $19(8.4)$ \\
Neck circumference/height index & $0.19 \pm 0.02$ \\
Waist/hip index & $0.78 \pm 0.07$ \\
Salient symptoms & $60(26.5)$ \\
Snoring n (\%) & $35(15.6)$ \\
Habitual snoring n (\%) & $7(3.1)$ \\
Reported sleep apnoeas n $(\%)$ & $68(30.1)$ \\
Excessive daytime sleepiness $\mathrm{n}(\%)$ & \\
Polygraphic findings & $539.2 \pm 67.7$ \\
TRT min & $297.2 \pm 118.2$ \\
TRT $\mathrm{sup}$ min & $97 \pm 0.6$ \\
Baseline $S \mathrm{a}, \mathrm{O}_{2} \%$ & $89.4 \pm 4.3$ \\
Sa, $\mathrm{O}_{2}$ nadir $\%$ & $0.15 \pm 0.63$ \\
TRT at $S \mathrm{a}, \mathrm{O}_{2}$ of $<90 \%$ & $1.55 \pm 1.97$ \\
ODI & $2.38 \pm 1.87$ \\
RDI & $15.9 \pm 11.4$ \\
Cardiac events &
\end{tabular}

Data are presented as mean \pm SD. BMI: body mass index; TRT: total recording time; TRTsup: TRT in the supine position; $\mathrm{Sa}_{2} \mathrm{O}_{2}$ : arterial oxygen saturation; ODI: oxygen desaturation index; RDI: respiratory disturbance index. \#: concurrent with respiratory event and/or oxygen desaturation. 
circumference/height index and waist/hip index in males compared to females, and excessive daytime sleepiness in females compared to males (table 2). The frequency of excessive daytime sleepiness was significantly higher in the postpubertal group than in the peripubertal group ( 37 versus $20 \%, \mathrm{p}=0.04$ ).

\section{Sex differences in the postpubertal group}

The postpubertal group included 136 subjects, 39.7\% male and $60.3 \%$ female, aged $16.3 \pm 1.7$ and $15.6 \pm 1.9 \mathrm{yrs}$, respectively. The study was carried out $3.3 \pm 1.1$ yrs after axillary hair development and peak height velocity in males, and $4.1 \pm 1.9$ yrs after menarche in females (range $2-8$ yrs). With regard to anthropometric variables, males compared to females exhibited significantly higher neck circumference/height index and waist/hip index (table 2). Conversely, the frequency of snoring was significantly higher in males, whereas headache was significantly higher in females. Results of cardiorespiratory polygraphy showed significant differences in favour of males, i.e. higher RDI, RDIsup, ODI and number of cardiac events and lower baseline $\mathrm{Sa}, \mathrm{O}_{2}$ and $\mathrm{Sa}, \mathrm{O}_{2}$ nadir (table 2). On ANCOVA, differences between males and females in RDI remained after controlling for age $(\mathrm{p}=0.019)$ and BMI $(p=0.045)$. However, after adjusting for waist/hip index, sex differences in RDI were not observed.

\section{Discussion}

The present results indicate that, in adolescents at advanced stages of sexual maturation, there are differences between males and females in the frequency of some symptoms related to SDB, such as snoring, as well as in the frequency and severity of cardiorespiratory polygraphy alterations. These features were not found among adolescents who were in an early phase of puberty.

It is well known that the clinical and polysomnographic characteristics of SDB are different in the various stages of life. In childhood, the mean prevalence of SDB is $\sim 2 \%$, without sex differences. In adulthood, population-based studies showed a prevalence of SDB that was higher in males than in females, particularly in the 30-60-yr stratum [22]. Some authors have recently shown an increase in the prevalence of SDB in females after the menopause. Since the mid-1980s, there has been progressive interest shown in the scientific literature regarding the effect of sex hormones on SDB, although results of early studies based on small study series were unclear [23, 24]. Recently, BIXLER et al. [14] provided epidemiological evidence for a lower prevalence of SDB in postmenopausal females using hormone replecement therapy than in nonusers. Studies in the elderly population revealed a high frequency of SDB, and it has been shown that sex differences in the prevalence of SDB decrease as age increases [6]. These findings support a protective effect of female sex hormones on some of the pathogenetic factors related to SDB. Conversely, increased testosterone levels have been associated with exacerbation or occurrence of obstructive sleep apnoea in a few adult male and female cases $[15,16]$. It has been speculated that testosterone may influence neuromuscular control of the upper airway and the central pattern of body fat distribution that have been related to SDB in adulthood.

In contrast to the evidence of the effect of age and sex on SDB in adult population groups, in the adolescent population, as far as the present authors are aware, the influence of sex hormones on sex differences in SDB has not been examined previously. With regard to methodological aspects, the strengths of the study include the participation of a general population sample of 226 adolescents aged 11-19 yrs and performance of overnight polygraphy in all subjects. For logistical purposes and to avoid refusals related to blood sample testing, arbitrary clinical criteria were used to classify subjects into the peripubertal and postpubertal groups [25-28], rather than serum hormone levels. This is a limitation of the study and should be taken into account in interpreting

Table 2.-Differences between males and females in the frequency distribution of anthropometric, clinical and polygraphic variables in the peripubertal and postpubertal adolescent groups

\begin{tabular}{|c|c|c|c|c|c|c|}
\hline & \multicolumn{3}{|c|}{ Peripubertal } & \multicolumn{3}{|c|}{ Postpubertal } \\
\hline & Males & Females & p-value & Males & Females & $\mathrm{p}$-value \\
\hline Subjects $n$ & 50 & 40 & & 54 & 82 & \\
\hline \multicolumn{7}{|l|}{ Anthropometric parameters } \\
\hline Age yrs & $13.5 \pm 1.2$ & $13.1 \pm 0.8$ & NS & $16.3 \pm 1.7$ & $15.6 \pm 1.9$ & 0.01 \\
\hline $\mathrm{BMI} \mathrm{kg} \cdot \mathrm{m}^{-2}$ & $21.3 \pm 3.8$ & $21.9 \pm 3.9$ & NS & $22.6 \pm 3.5$ & $21.6 \pm 3.3$ & NS \\
\hline Obesity n $(\%)$ & $3(6.1)$ & $4(10.0)$ & NS & $6(11.1)$ & $6(7.3)$ & NS \\
\hline Neck circumference/height index & $0.20 \pm 0.01$ & $0.19 \pm 0.003$ & 0.01 & $0.20 \pm 0.01$ & $0.19 \pm 0.01$ & 0.001 \\
\hline Waist/hip index & $0.85 \pm 0.06$ & $0.76 \pm 0.05$ & 0.000 & $0.80 \pm 0.06$ & $0.73 \pm 0.04$ & 0.000 \\
\hline \multicolumn{7}{|l|}{ Salient symptoms } \\
\hline Habitual snoring n $(\%)$ & $8(16.0)$ & $7(17.5)$ & NS & $12(22.2)$ & $8(9.9)$ & 0.04 \\
\hline Reported sleep apnoeas n (\%) & $1(2.3)$ & $4(12.5)$ & NS & $1(2.8)$ & $1(2.0)$ & NS \\
\hline Excessive daytime sleepiness n (\%) & $6(12)$ & $12(30)$ & 0.03 & $20(37)$ & $30(37)$ & NS \\
\hline \multicolumn{7}{|l|}{ Polygraphic findings } \\
\hline TRT min & $549.3 \pm 50.4$ & $541.7 \pm 59.0$ & NS & $554.2 \pm 73.3$ & $522.1 \pm 73.7$ & NS \\
\hline Baseline $\mathrm{Sa}_{\mathrm{a}} \mathrm{O}_{2} \%$ & $96.8 \pm 0.7$ & $97.1 \pm 0.7$ & NS & $96.9 \pm 0.7$ & $97.1 \pm 0.6$ & 0.05 \\
\hline $\mathrm{Sa}_{\mathrm{a}} \mathrm{O}_{2}$ nadir $\%$ & $88.9 \pm 3.6$ & $89.0 \pm 3.7$ & NS & $88.5 \pm 3.6$ & $90.4 \pm 5.3$ & 0.02 \\
\hline TRT at $S \mathrm{a}, \mathrm{O}_{2}$ of $<90 \%$ & $0.11 \pm 0.25$ & $0.08 \pm 0.21$ & NS & $0.17 \pm 0.40$ & $0.19 \pm 0.97$ & NS \\
\hline ODI & $1.56 \pm 2.27$ & $1.67 \pm 2.00$ & NS & $1.96 \pm 1.86$ & $1.21 \pm 1.77$ & 0.02 \\
\hline RDI & $2.69 \pm 1.69$ & $2.34 \pm 1.58$ & NS & $2.74 \pm 1.90$ & $1.98 \pm 1.95$ & 0.03 \\
\hline RDIsup & $4.88 \pm 2.97$ & $4.51 \pm 3.82$ & NS & $7.13 \pm 6.46$ & $4.30 \pm 4.86$ & 0.008 \\
\hline Cardiac events $\#$ & $18.80 \pm 14.60$ & $18.27 \pm 12.00$ & NS & $17.84 \pm 9.50$ & $11.82 \pm 8.50$ & 0.000 \\
\hline
\end{tabular}

Data are presented as mean \pm SD. BMI: body mass index; TRT: total recording time; $\mathrm{Sa}, \mathrm{O}_{2}$ : arterial oxygen saturation; ODI: oxygen desaturation index; RDI: respiratory disturbance index; RDIsup: RDI in the supine position; NS: nonsignificant. \#: concurrent with respiratory event and/or oxygen desaturation. 
the results. Another methodological aspect that should be emphasised is that, rather than employing nasal prongs or an intraoesophageal catheter to monitor airflow, a thermistor was used. This does not invalidate the differences that were found in respiratory events between the two groups, although it is possible that some respiratory events associated with flow limitation might not have been detected.

Of the adolescents, $\sim 15 \%$ were considered habitual snorers. This figure is similar to that reported by others for paediatric groups and student populations [18, 19, 29, 30]. Sex differences in the peripubertal group were not observed, but the frequency of habitual snorers was significantly higher among males than among females in the postpubertal group. These results may indicate that well-established clinical differences in adult subjects become evident at puberty. The frequency of daytime hypersomnolence was significantly higher in females than in males in the peripubertal group, whereas sex differences in excessive daytime sleepiness in the postpubertal group were not found. However, it is known that the cause of daytime hypersomnolence in adolescents is multifactorial [31, 32]. Adolescence delays the "sleep-on" setting in the biological clock with a trend towards going to bed and waking up late, which cannot be accomplished within the scholarly timetable, and results in a cumulative sleep debt, leading to adolescent daytime sleepiness.

Data regarding respiratory pattern in general population samples of adolescents are lacking, and, in most series, broader age ranges are included, subjects are selected according to certain characteristics, such as obesity, or polygraphy is only carried out on a subset of the participants [33, 34]. Sex differences in the peripubertal group regarding polygraphic alterations were not observed. However, a higher number of respiratory events on polygraphy were observed in postpubertal males than females. The same trend was observed for oximetric parameters. Sex differences in polygraphic alterations in the postpubertal group were not due to age or BMI, but differences disappeared after adjusting for waist/hip index, which indicates that body fat distribution, assessed by the waist-to-hip ratio, may have had an effect on the results obtained. However, it seems likely that, in adolescents in advanced stages of pubertal development, other anatomical factors, such as craniofacial alterations or nasal obstruction, which could be present from childhood, or functional ones, may account for the lower pharyngeal collapsibility among females.

In summary, postpubertal adolescents showed sex differences in clinical and polygraphic parameters related to sleepdisordered breathing that were not observed at earlier pubertal stages. These findings, which need to be confirmed in longitudinal studies, support the influence of sex hormones on sex differences in sleep-disordered breathing that seem to become apparent at the time of sexual maturation.

Acknowledgements. The authors would like to thank M. Pulido for editing the manuscript and editorial assistance.

\section{References}

1. Masa JF, Rubio M, Findley LJ. Habitually sleepy drivers have a high frequency of automobile crashes associated with respiratory disorders during sleep. Am J Respir Crit Care Med 2000; 162: 1407-1412.

2. Nieto FJ, Young TB, Lind BK. Association of sleepdisordered breathing, sleep apnea, and hypertension in a large community-based study: Sleep Heart Health Study. JAMA 2000; 283: 1829-1836.
3. Peppard P, Young T, Palta M, Skatrud J. Prospective study of the association between sleep-disordered breathing and hypertension. N Engl J Med 2000; 342: 1378-1384.

4. Block AJ, Boysen PG, Wynne JW, Hunt LA. Sleep apnea, hypopnea, and oxygen desaturation in normal subjects. $N$ Engl J Med 1979; 300: 513-517.

5. Guilleminault C, Quera-Salva MA, Partinen M, Jamieson A. Women and the obstructive sleep apnea syndrome. Chest 1988; 93: 104-109.

6. Bixler EO, Vgontzas AN, Ten Have T, Tyson K, Kales A. Effects of age on sleep apnoea in men: I. Prevalence and severity. Am J Respir Crit Care Med 1998; 157: 144-148.

7. Young T, Palta M, Dempsey J, Skatrud J, Weber S, Badr S. The occurrence of sleep-disordered breathing among middleaged adults. $N$ Engl J Med 1993; 328: 1230-1235.

8. Durán J, Esnaola S, Rubio R, Iztueta A. Obstructive sleep apnea-hypopnea and related clinical features in a population-based sample of subjects aged 30 to $70 \mathrm{yr}$. Am J Respir Crit Care Med 2001; 163: 685-689.

9. Chervin RD. Sleepiness, fatigue, tiredness, and lack of energy in obstructive sleep apnea. Chest 2000; 118: 372-379.

10. Young T, Hutton R, Finn L, Badr S, Palta M. The gender bias in sleep apnea diagnosis. Are women missed because they have different symptoms? Arch Intern Med 1996; 156: 2445-2451.

11. Mohsenin V. Gender differences in the expression of sleepdisordered breathing. Role of upper airway dimensions. Chest 2001; 120: 1442-1447.

12. Pillar G, Malhotra A, Fogel R, Beauregard J, Schnall R, White D. Airway mechanics and ventilation in response to resistive loading during sleep: influence of gender. $\mathrm{Am} \mathrm{J}$ Respir Crit Care Med 2000; 162: 1627-1632.

13. Popovic R, White D. Upper airway muscle activity in normal women: influence of hormonal status. J Appl Physiol 1998; 84: 1055-1062.

14. Bixler EO, Vgontzas AN, Lin HM, et al. Prevalence of sleepdisordered breathing in women. Effects of gender. $\mathrm{Am} \mathrm{J}$ Respir Crit Care Med 2001; 163: 608-613.

15. Dexter DD, Dovre EJ. Obstructive sleep apnea due to endogenous testosterone production in a woman. Mayo Clin Proc 1998; 73: 246-248.

16. Cistulli PA, Grunstein RR, Sullivan CE. Effect of testosterone administration on upper airway collapsibility during sleep. Am J Respir Crit Care Med 1994; 149: 530-532.

17. Rosen C, D'Andrea L, Haddad G. Adult criteria for obstructive sleep apnea do not identify children with serious obstruction. Am Rev Respir Dis 1992; 146: 1231-1234.

18. Teculescu D, Caillier I, Perrin P, Rebstock E, Rauch A. Snoring in French preschool children. Pediatr Pulmonol 1992; 13: 239-244.

19. Gaultier C. Obstructive sleep apnea in infants and children: established facts and unsettled issues. Thorax 1995; 50: 1204 1210.

20. Guilleminault C, Partinen M, Praud JP, Quera-Salva MA, Powell N, Riley R. Morphometric facial changes and obstructive sleep apnea in adolescents. J Pediatrics 1989; 114: 997-999.

21. Sánchez-Armengol A, Fuentes-Pradera MA, Capote-Gil F, et al. Sleep-related breathing disorders in adolescents aged 12 to 16 years. Clinical and polygraphic findings. Chest 2001; 119: 1393-1400.

22. Young T, Peppard PE, Gottlieb DJ. Epidemiology of obstructive sleep apnea. A population health perspective. Am J Respir Crit Care Med 2002; 165: 1217-1239.

23. Cistulli PA, Barnes DJ, Grunstein RR, Sullivan CE. Effect of short-term hormone replacement in the treatment of obstructive sleep apnoea in postmenopausal women. Thorax 1994; 49: 699-702.

24. Pickett CK, Regensteiner JG, Woodard WD, Hagerman DD, Weil JV, Moore LG. Progestin and estrogen reduce sleep-disordered breathing in postmenopausal women. J Appl Physiol 1989; 66: 1656-1661. 
25. Cortes Blanco A, Ferrandez Longas A, Mayayo Dehesa E, Labarta Aizpun JI. Valores de referencia de FSH, LH, testosterona total, testosterona libre, $17-\beta$-estradiol y SHBG en niños sanos zaragozanos. An Esp Pediatr 1999; 51: 159-166.

26. Guvenc H, Guvenc M, Berki R. Serum gonadotropins and estradiol levels in Turkish pubertal girls. Indian $J$ Pediatr 1990; 57: 421-423.

27. Kulik-Rechberger B, Rechberger T. Leptin as a trigger for puberty in girls. Ginekol Pol 2001; 72: 535-540.

28. Fechner PY. Gender differences in puberty. $J$ Adolesc Health 2002; 30: 4 Suppl. 1, 44-48.

29. Redline S, Tishler PV, Schluchter M, Aylor J, Clark K, Graham G. Risk factors for sleep-disordered breathing in children. Associations with obesity, race, and respiratory problems. Am J Respir Crit Care Med 1999; 159: 1527-1532.
30. Hui D, Chan J, Ho A, Choy D, Lai C, Leng R. Prevalence of snoring and sleep-disordered breathing in a student population. Chest 1999; 116: 1530-1536.

31. Carskadon M, Vieira C, Acebo C. Association between puberty and delayed phase preference. Sleep 1993; 16: 258262.

32. Gau SF, Soong WT. Sleep problems of junior high school students in Taipei. Sleep 1995; 18: 667-673.

33. Acebo C, Millman RP, Rosenberg C, Cavallo A, Carskadon M. Sleep, breathing, and cephalometrics in older children and young adults. Part I - Normative values. Chest 1996; 109: 664-672.

34. Marcus C, Omlin K, Basinki D, et al. Normal polysomnographic values for children and adolescents. Am Rev Respir Dis 1992; 146: 1235-1239. 